\title{
Lexis
}

Journal in English Lexicology

Book reviews | 2019

\section{Beate HAMPE (ed.), Metaphor. Embodied Cognition and Discourse}

Cambridge University Press, 2017, 392 pages

\section{Liana Pop}

\section{OpenEdition}

Journals

Édition électronique

URL : http://journals.openedition.org/lexis/2606

DOI : $10.4000 /$ lexis. 2606

ISSN : 1951-6215

Éditeur

Université Jean Moulin - Lyon 3

Référence électronique

Liana Pop, «Beate HAMPE (ed.), Metaphor. Embodied Cognition and Discourse », Lexis [En ligne], Recensions, mis en ligne le 21 janvier 2019, consulté le 25 septembre 2020. URL : http:// journals.openedition.org/lexis/2606; DOI : https://doi.org/10.4000/lexis.2606

Ce document a été généré automatiquement le 25 septembre 2020.

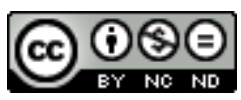

Lexis is licensed under a Creative Commons Attribution-NonCommercial-NoDerivatives 4.0 International License. 


\section{Beate HAMPE (ed.), Metaphor. Embodied Cognition and Discourse}

Cambridge University Press, 2017, 392 pages

\section{Liana Pop}

\section{RÉFÉRENCE}

Beate Hampe

Metaphor. Embodied Cognition and Discourse, Cambridge University Press, 2017. ISBN :

978-1-107-19833-3, Prix : 95£, 392 pages

1 Ce volume, édité par les soins de Beate Hampe de l'Université allemande d'Erfurt, rassemble les recherches effectuées par plusieurs spécialistes de la métaphore, lors d'un projet dédié à la réunification de visions opposées dans le domaine. Pour les moins avisés, précisons qu'il s'agit d'études qui se sont inscrites dans la théorie standard de la métaphore cognitive (CMT) élaborée en 1980 par Lakoff et Johnson dans leur ouvrage Metaphors We Live By, en la développant ou, de façon plus ou moins radicale, en la remettant en cause. Les démarches dans ce recueil viennent d'horizons divers: linguistique, pragmatique, cognition, biochimie, neurologie, psychologie (psycholinguistique et psychologie cognitive), philosophie, études culturelles, sciences de la communication, linguistique appliquée, média. L'enjeu va, bien sûr, au-delà de celui de la CMT, dont la révolution consistait à remettre en question l'appartenance linguistique de la métaphore, la situer d'abord dans l'esprit et l'ancrer essentiellement dans le corps et l'expérience humaine avec le corps, selon un schéma de transfert sémantique source-cible. Or, cette vision, trop statique et trop réductive, avec des exemples hors contexte, n'a pas tardé à être attaquée par les tenants d'une observation directe du discours et des interactions situées, verbales et/ou non verbales. La notion initiale de métaphore «incorporée » (embodied metaphor) se verra ainsi remplacée par celle de « métaphoricité », vue comme activité discursive, négociation interpersonnelle, 
sociale et culturelle des degrés de métaphoricité. Ainsi, on ne se demande plus ce qu'est la métaphore, mais ce qu'elle fait (p. 22).

Ces ajustements de la théorie de départ se trouvent synthétisés par la coordinatrice du volume dans son Introduction, et non moins réaffirmées dans l'Épilogue, dans un esprit fortement conciliateur et fédérateur, par un des représentants les plus connus des théories contemporaines de la cognition: Raymond W. Gibbs, auteur du célèbre ouvrage publié en 1994, Poetics of Mind (Poétique de l'esprit, en français).

Le livre regroupe les contributions divisées en quatre parties, et organisées suivant leur apport thématique : Metaphor in Cognition (I), More than Metaphor (II), Metaphor in Discourse (III) et Salient Metaphor (IV).

Dans le premier volet, une idée forte fait son chemin, qui va se développer dans plusieurs approches - celle que les métaphores premières vont s'avérer souvent des points de départ pour des conceptualisations plus riches (Joseph E. Grady et Giorgio Ascoli, p. 37). En tant que perspective neuronale et biochimique, cette idée est soutenue ici par l'évidence de "cartes corticales des expériences-sources» (scénarios), plus stables et identifiables que les «cartes-cibles reliées » (goal related maps), ces dernières censées être plus diffuses dans le cerveau. L'observation a été menée sur trois métaphores cognitives catégorisant l'abstrait par le concret (la difficulté par la lourdeur, l'élément dominant par un élément placé à une haute position, et le bonheur par la clarté), mais exigerait, d'après les auteurs mêmes, à être développée.

5 Pour la psychologie cognitive qui procède souvent de façon expérimentale (contribution de Daniel Casasanto), il s'avère que la conceptualisation (du temps par l'espace, par exemple) peut varier chez divers sujets en fonction de leur vécu ou de leur culture, ce qui oblige à prendre en compte moins une universalité (point de vue de la CMT), qu'une flexibilité des métaphores et un dynamisme des vies mentales. Quant à Yeshayahu Shen et Roy Porat, ils vont mettre en cause l'unidirectionnalité des schémas conceptuels source-cible soutenue par la théorie de l'incorporation, en proposant néanmoins une vision concessive vis-à-vis de la CMT : leur hypothèse est celle d'une bidirectionnalité des métaphores premières au niveau pré-conceptuel de leur formation, suivie, sous l'influence du langage, par une phase directionnelle. L'existence de métaphores inversées est ainsi proposée et démontrée par de nouveaux éléments venant enrichir la théorie.

D'un autre côté, sans contester l'influence directe du corps sur la cognition (et, partant, la notion de «métaphore incorporée»), Valentine Cuccio va plus loin, elle aussi, en distinguant deux rôles et, deux niveaux d'incorporation: l'un, plus profond, dit «invisible» (Body Schema), celui des habiletés de perception sensori-motrice, s'actualisant par des métonymies située, elles, en dehors des représentations du corps ; l'autre, dit niveau «visible » (Body Image), issu d'une représentation mentale du corps en termes métaphoriques.

7 À cette perspective « enrichie » de la métaphore cognitive contribuent également Bodo Winter et Teenie Matlock qui, exemples à l'appui, vont prouver que les métaphores premières ne sont pas des entités conceptuelles pures, mais des phénomènes multiniveaux, produits aussi en dehors du corps (langage, gestes, culture) et pouvant se combiner entre eux.

8 En conclusion, l'apport de cette première partie dédiée à la métaphore dans la cognition est évident, permettant d'affirmer non seulement que la théorie pionnière de 
Lakoff et Johnson a bel et bien éveillé l'intérêt bien au-delà des frontières (États-Unis, Israël, Italie, Angleterre) et au-delà des disciplines, mais aussi qu'elle a acquis, entre autres, des appuis neurologiques et qu'elle a évolué en transgressant et élargissant ses cadres et objets d'analyse. L'apport des neurosciences, de la biochimie et de la psychologie à cette théorie de l'incorporation du sens semble avoir changé la donne d'une vision de départ purement sémantique.

9 Le deuxième volet du livre va étoffer considérablement la catégorie de la métaphore en la complexifiant et la dynamisant. C'est ce qu'annonce le titre de cette section - «Plus que des métaphores» (More than Metaphor) - où la métaphore se verra attribuer, à la place de schémas orientés vers l'objet, des schémas-cadres d'actions incorporées.

10 Ainsi, le cas examiné par Irene Mittelberg et Gina Joue - les gestes conceptualisant l'espace dans des discours académiques et des films - décrit-il la façon dont les gestes sont à même d'évoquer des métaphores, des métonymies et des cadres d'événements prototypiques. Par la notion de schémas mimétiques, la voie vers les recherches multimodales est ouverte, étoffant, au niveau gestuel, les modes d'interaction déjà observés entre la métonymie et la métaphore. Comme les gestes par lesquels, pour le déplacement d'un OBJET imaginaire, les locuteurs évoquent des cadres actionnels basiques tels les actions-source de POUSSER et ARRIVER DANS et la cible du CONTAINER.

11 Francisco J. Ruiz de Mendoza Ibáñez observe le même tandem métaphore-métonymie, phénomène recouvrant en fait des opérations cognitives plus basiques, qui interfèrent, se combinent et interagissent, construisant des schémas complexes, issus d'associations métaphoriques plus élémentaires. Agissant en associations, la sélection, l'abstraction, la substitution, l'intégration, etc. donnent lieu à des figures comme l'hyperbole, l'ironie, les métaphtonymes, les chaînes métonymiques ou métaphoriques, les amalgames et blends métaphoriques. L'avancée qu'apporte cette analyse est extrêmement dense et importante, avec, également, des "parcours » créatifs dessinés en configurations complexes pour chacun de ces cas de «figures", moins étudiés dans le domaine du cognitivisme.

12 Jeanette Littlemore prend pour exemple deux cas particuliers de métonymies : celle du type LA PARTIE DU CORPS POUR LA FONCTION, telle la tête pour donner des ordres, et celle conceptualisant les positions sociales, du type : LE HAUT POUR LE CHEF, LE BAS POUR LES OUVRIERS. Par sa vision, elle entend élargir, dynamiser et, ainsi, développer la notion de «cognition incorporée » en la situant en égale mesure dans l'environnement et la société avec ses usages, expliquant ainsi certains exemples par leur étymologie. L'approche exige l'utilisation par l'auteure de termes innovants, comme ceux de " cognition à incorporation sociale» (social embodied cognition, p. 167) ou de « cognition habillée » (enclothed cognition, p. 168).

13 Le bilan de cette section est consistant et va, effectivement, au-delà des simples métaphores, vu leur enrichissement avec des approches multimodales, des schémas beaucoup plus élaborés et une terminologie élargie.

14 Le volet dédié aux approches discursives (Metaphor in Discourse) s'avère encore plus intéressant, innovant et productif, la métaphore étant observée dans son instabilité, son dynamisme et sa transformation telle qu'utilisée par les locuteurs ou les scripteurs dans plusieurs types de discours. 

« carte cancer ». Sur ce forum, la communauté d'internautes (personnes atteintes du cancer) s'exprime souvent avec des termes plus légers, voire comiques, issus de la métaphore conceptuelle LA VIE EST UN JEU DE CARTES. La métaphore et l'humour, dans des emplois très flexibles, plus ou moins littérales par endroits, semblent remplir plusieurs fonctions discursives et sociales, parfois communes, dont la solidarité, l'esprit de groupe, le relâchement des tensions, etc. Mais pour le chercheur, ce qui intéresse dans le fonctionnement de cette métaphore, ce sont notamment ses manifestations d'instabilité et les zones de semi-littéralisation du terme, ainsi qu'une certaine transgression des genres (contamination avec les genres publicitaires ou autres). Le terme de "métaphorème" est proposé pour recouvrir ce comportement flexible, agissant aussi comme attracteur sémantique pour une communauté donnée. discursif - est repris par Alice Deignan dans l'observation d'images et de textes narratifs (à l'oral, dans les dessins et les photographies) issus de la métaphore LA VIE EST UN VOYAGE, ou d'une de ses versions : UNE THESE DE DOCTORAT EST UN VOYAGE. Dans le registre graphique et la photographie, les correspondances relevées sont: le beau et le mauvais temps pour les états d'âme, les obstacles pour les difficultés, les montées et les descentes d'un chemin pour les phases plus ou moins créatives, les croisements de chemins pour les choix à faire, etc. L'idée qui est ici développée est que les métaphores arrivent à déclencher des séquences narratives que les utilisateurs peuvent facilement développer, créant des correspondances métaphoriques de façon plus ou moins inventive. avatars possibles des métaphores : les " récits métaphoriques " (metaphorical stories) allégories, bandes dessinées, le roman orwellien La ferme des animaux, etc. - et les "métaphores narratives» (story metaphors) - celles qui peuvent déclencher des récits. Le problème des métaphores complexes est également posé, avec, pour exemples, des objets symboliques, comme la Statue de la Liberté à New York et celle de la Justice sur les tribunaux, artefacts dont les différents éléments renvoient à des symboles reconnus comme conventionnels. métaphores structurelles de Lakoff et Johnson ne sont point des schémas aussi basiques qu'elles étaient imaginées hors contexte, mais, au contraire, des combinaisons de plusieurs métaphores premières, enrichies situationnellement et culturellement par l'usage - objets d'interprétation variables de la part des utilisateurs. Son observation concerne la zone dynamique des films d'animation. Enfin, la phrase finale de l'auteur vient reformuler radicalement le premier titre de la théorie CMT : «Incorporées et supportées par un contexte culturel incroyablement riche, elles [les métaphores] deviennent les métaphores de notre vie quotidienne" (Embedded in and supported by incredible rich cultural contexts, these become the metaphors we actually live by. p. 256).

Cette partie du volume dédiée à la métaphore dans le discours apporte une autre contribution intéressante, celle de Thomas W. Jensen, qui observe le comportement de la métaphore dans des textes oraux, spontanés (réunions d'affaires, thérapies de couple). Il constate que, dans la production des discours, la métaphoricité (l'acte de faire des métaphores) se manifeste par des reprises et développements par les interlocuteurs des aspects «durs» et/ou périphériques des métaphores, en co- 
réflexion et co-action. Et de conclure que « la cognition (ou les habiletés cognitives) nous ne la possédons pas, mais nous la construisons pendant notre activité exploratrice de production du sens " (we do not [...] possess cognition (or cognitive abilities), instead we do cognition in our active and explorative sens-making, p. 261). La représentation dynamique, flexible et textuelle de la métaphore est ainsi dessinée dans ce grand volet, avec des études observant différents types de communication naturelle, artistique ou virtuelle : textes, dessins, bandes dessinées, sculptures, films, etc.

Enfin, deux chapitres sont proposés dans la dernière partie intitulée Salient Metaphor, les deux concernant les métaphores délibérées vs. non délibérées, ou autrement dit, les métaphores classiquement appelées " vives » (selon Ricoeur) vs. celles dites «mortes » ou « endormies ». Une observation intéressante est ici menée sur un corpus multimodal - un cours de danse, activant parole, gestes et mouvements corporels. L'alternance de ces éléments communicatifs destinés à montrer les diverses façons de bouger font surgir et se combiner des métaphores verbales et non verbales avec plus ou moins de force, dans un travail de métaphorisation très complexe.

21 Raymond W. Gibbs clôture cet énorme parcours de recherches et de perspectives, ces apports considérables, parfois convergents et renforçant la théorie de départ en sémantique cognitive, parfois plutôt divergents et prenant des chemins pragmatiques, en adoptant une perspective multimodale, sociale et culturelle. Ce qu'il suggère comme directions à prendre pour l'avenir, c'est une fédération des deux visions principales celle de la métaphore incorporée et celle de la métaphore discursive - dans leurs interrelations à plusieurs niveaux. D'après l'auteur, les principes émergents qui semblent avoir déjà œuvré pour une telle harmonisation seraient la nature incorporée déjà prouvée de la métaphore discursive, ainsi que le contexte social pris en considération pour toutes les actions incorporées, que ce soit d'un point de vue organique ou conceptuel. Ses recommandations pour rendre compatibles les recherches sont, d'une part, d'éviter de rejeter, dans une attitude de "solipsisme méthodologique » (p. 328) les autres perspectives ou niveaux d'analyse, et d'autre part, de "lire les autres" (cette remarque laisse exprimer ici un étonnement quant à l'absence de renvois au moins minimaux, dans les approches de ce livre, à des noms incontournables, comme Roche, Culioli, Wierzbicka, Ricoeur, à des notions existant depuis longtemps en rhétorique - comme celle de "métaphore filée ", dynamique et discursive par excellence - ou à des ressources classiques en analyse sémantique - les incursions étymologiques et les mécanismes de changements de sens, trop vaguement invoquées dans les recherches de ce volume). Gibbs impose comme objet de recherche ce qu'il faudrait dorénavant appeler actions métaphoriques de toutes sortes, à observer à des niveaux complexes et dynamiques de manifestation. L'objectif impératif serait de " créer des théories de l'action métaphorique capable de rendre compte de ce que nous savons sur les manières réelles d'agir des gens dans le monde réel » (The imperative is to create theories of metaphorical action that take full acount of what we know about how people really work in the real world, p. 334).

Ce volume montre, sans conteste, l'énorme travail entrepris par des chercheurs venant de tous horizons et qui ont senti et voulu relever le défi lancé par Lakoff et Johnson (dans leur version de Metaphors We Live By de 2003), c'est-à-dire aborder « des méthodes d'investigation collaboratives et transdisciplinaires " (this book [Metaphors We Live By] remains just as controversial and radical today as when it first appeared. It calls into question business as usual and requires new collaborative cross-disciplinary methods of inquiry; Lakoff \& 
Johnson 2003: 274). Les quatre décennies de leur première version ont bien porté leurs fruits.

\section{BIBLIOGRAPHIE}

GIBBS Raymond W. Jr.,1994, Poetics of Mind. Figurative Thought, Language and Understanding, Cambridge: Cambridge University Press.

LAKOFF George, JOHNSON Mark, 2003 (1980), Metaphors We Live By, London: The University of Chicago press.

\section{AUTEURS}

\section{LIANA POP}

Liana Pop est Professeur émérite à la Faculté des Lettres de l'Université Babeş-Bolyai de Cluj (Roumanie), et est spécialiste, notamment, d'analyse du discours et de l'oral. Un de ses cours de niveau doctoral propose comme thématique la conceptualisation en langue et en linguistique. Auteur de nombreuses méthodes d'enseignement du français et du roumain langues étrangères (v. Guide du Roumain, chez Assimil, 2014), elle a aussi dirigé des collections spécialisées en linguistique et langues aux Éditions Echinox de Cluj. Deux de ses théories du discours ont été publiées aux Éditions Peeters (Espaces discursifs, Paris, Louvain, 2000) et Peter Lang (La grammaire graduelle, à une virgule près, Bern, Berlin, New York, 2005). La traduction en roumain du Dictionnaire encyclopédique de pragmatique, Seuil, 1994 a été co-dirigée par L. Pop, qui a aussi entrepris la traduction de deux autres livres de linguistique française : La pragmatique aujourd'hui, de A. Reboul et J. Moeschler, Éditions du Seuil, et l'Introduction à la linguistique contemporaine, d'A. Auchlin et J. Moeschler, Éditions A. Colin. Elle a participé à plusieurs projets de langue et de linguistique, comme directrice, membre ou expert. 